\title{
NON-RIEMANNIAN GRAVITATIONAL INTERACTIONS
}

\author{
ROBIN W. TUCKER and CHARLES WANG \\ School of Physics and Chemistry, Lancaster University \\ Lancaster LA1 $4 Y B, U . K$. \\ E-mail:r.tucker@lancaster.ac.uk,c.wang@lancaster.ac.uk
}

\begin{abstract}
Recent developments in theories of non-Riemannian gravitational interactions are outlined. The question of the motion of a fluid in the presence of torsion and metric gradient fields is approached in terms of the divergence of the Einstein tensor associated with a general connection. In the absence of matter the variational equations associated with a broad class of actions involving non-Riemannian fields give rise to an Einstein-Proca system associated with the standard Levi-Civita connection.
\end{abstract}

1. Introduction. Einstein's theory of gravity has an elegant formulation in terms of (pseudo-) Riemannian geometry. The field equations follow as a local extremum of an action integral under metric variations. In the absence of matter the integrand of this action is simply the curvature scalar associated with the curvature of the Levi-Civita connection times the (pseudo-) Riemannian volume form of spacetime. Such a connection $\nabla$ is torsion-free and metric compatible. Thus for all vector fields $X, Y$ on the spacetime manifold, the tensors given by:

$$
\begin{gathered}
\mathbf{T}(X, Y)=\nabla_{X} Y-\nabla_{Y} X-[X, Y] \\
\mathbf{S}=\nabla \mathbf{g}
\end{gathered}
$$

are zero, where $\mathbf{g}$ denotes the metric tensor, $\mathbf{T}$ the $2-1$ torsion tensor and $\mathbf{S}$ the gradient tensor of $\mathbf{g}$ with respect to $\nabla$. Such a Levi-Civita connection provides a useful reference connection since it depends entirely on the metric structure of the manifold.

Non-Riemannian geometries feature in a number of theoretical descriptions of the interactions between fields and gravitation. Since the early pioneering work by Weyl, Cartan, Schroedinger and others such geometries have often provided a succinct and elegant guide towards the search for unification of the forces of nature [1]. In recent times interactions with supergravity have been encoded into torsion fields induced by spinors and dilatonic interactions from low energy effective string theories have been

1991 Mathematics Subject Classification: Primary 83D05.

The paper is in final form and no version of it will be published elsewhere. 
encoded into connections that are not metric-compatible $[2,3,4,5]$. However theories in which the non-Riemannian geometrical fields are dynamical in the absence of matter are more elusive to interpret. It has been suggested that they may play an important role in certain astrophysical contexts [6]. Part of the difficulty in interpreting such fields is that there is little experimental guidance available for the construction of a viable theory that can compete effectively with general relativity in domains that are currently accessible to observation. In such circumstances one must be guided by the classical solutions admitted by theoretical models that admit dynamical non-Riemannian structures $[7,8,9,10,11$, $17,16]$. This approach is being currently pursued by a number of groups $[6,12,13,14$, 15, 17]. An associated problem concerns the role of the torsion and metric gradients in determining the motion of matter $[18,19,20]$. This paper briefly reports on recent efforts to address these questions.

2. Non-Riemannian geometry. A linear connection $\nabla$ on a manifold provides a covariant way to differentiate tensor fields. It provides a type preserving derivation on the algebra of tensor fields that commutes with contractions. Given an arbitrary local basis of vector fields $\left\{X_{a}\right\}$ the most general linear connection is specified locally by a set of $n^{2} 1$-forms $\Lambda_{b}^{a}$ where $n$ is the dimension of the manifold:

$$
\nabla_{X_{a}} X_{b}=\Lambda_{b}^{c}\left(X_{a}\right) X_{c}
$$

Such a connection can be fixed by specifying a $(2,0)$ symmetric metric tensor $\mathbf{g}$, a (2-antisymmetric, 1$)$ tensor $\mathbf{T}$ and a $(3,0)$ tensor $\mathbf{S}$, symmetric in its last two arguments. If we require that $\mathbf{T}$ be the torsion of $\nabla$ and $\mathbf{S}$ be the gradient of $\mathbf{g}$ then it is straightforward to determine the connection in terms of these tensors. Indeed since $\nabla$ is defined to commute with contractions and reduce to differentiation on scalars it follows from the relation

$$
X(\mathbf{g}(Y, Z))=\mathbf{S}(X, Y, Z)+\mathbf{g}\left(\nabla_{X} Y, Z\right)+\mathbf{g}\left(Y, \nabla_{X} Z\right)
$$

that

$$
\begin{aligned}
2 \mathbf{g}\left(Z, \nabla_{X} Y\right)= & X(\mathbf{g}(Y, Z))+Y(\mathbf{g}(Z, X))-Z(\mathbf{g}(X, Y)) \\
& -\mathbf{g}(X,[Y, Z])-\mathbf{g}(Y,[X, Z])-\mathbf{g}(Z,[Y, X]) \\
& -\mathbf{g}(X, \mathbf{T}(Y, Z))-\mathbf{g}(Y, \mathbf{T}(X, Z))-\mathbf{g}(Z, \mathbf{T}(Y, X)) \\
& -\mathbf{S}(X, Y, Z)-\mathbf{S}(Y, Z, X)+\mathbf{S}(Z, X, Y)
\end{aligned}
$$

where $X, Y, Z$ are any vector fields. The general curvature operator $\mathbf{R}_{X, Y}$ defined in terms of $\nabla$ by

$$
\mathbf{R}_{X, Y} Z=\nabla_{X} \nabla_{Y} Z-\nabla_{Y} \nabla_{X} Z-\nabla_{[X, Y]} Z
$$

is also a type-preserving tensor derivation on the algebra of tensor fields. The general (3, 1) curvature tensor $\mathbf{R}$ of $\nabla$ is defined by

$$
\mathbf{R}(X, Y, Z, \beta)=\beta\left(R_{X, Y} Z\right)
$$

where $\beta$ is an arbitrary 1 -form. This tensor gives rise to a set of local curvature 2 -forms $R^{a}{ }_{b}$ :

$$
R_{b}^{a}(X, Y)=\frac{1}{2} \mathbf{R}\left(X, Y, X_{b}, e^{a}\right)
$$


where $\left\{e^{c}\right\}$ is any local basis of 1 -forms dual to $\left\{X_{c}\right\}$. In terms of the contraction operator $i_{X}$ with respect to $X$ one has $i_{X_{b}} e^{a} \equiv i_{b} e^{a}=e^{a}\left(X_{b}\right)=\delta^{a}{ }_{b}$. In terms of the connection forms

$$
R_{b}^{a}=\mathrm{d} \Lambda_{b}^{a}+\Lambda_{c}^{a} \wedge \Lambda_{b}^{c} .
$$

In a similar manner the torsion tensor gives rise to a set of local torsion 2-forms $T^{a}$ :

$$
T^{a}(X, Y) \equiv \frac{1}{2} e^{a}(\mathbf{T}(X, Y))
$$

which can be expressed in terms of the connection forms as

$$
T^{a}=\mathrm{d} e^{a}+\Lambda_{b}^{a} \wedge e^{b} .
$$

Since the metric is symmetric the tensor $\mathbf{S}$ can be used to define a set of local non-metricity 1-forms $Q_{a b}$ symmetric in their indices:

$$
Q_{a b}(Z)=\mathbf{S}\left(Z, X_{a}, X_{b}\right) .
$$

3. The motion of a charged fluid. The traditional Einstein-Maxwell equations for an electrically charged ideal fluid with mass density $\rho$ (and composed of elements with charge to mass ratio $\frac{e}{m}$ ) are:

$$
\begin{gathered}
\operatorname{Ein}=\boldsymbol{\tau} \\
\mathrm{d} * F=j \\
\mathrm{~d} F=0
\end{gathered}
$$

where $\mathbf{E i n}=\mathbf{R i c}-\frac{1}{2} \mathbf{g} \mathcal{R}$ is the Einstein tensor associated with the Levi-Civita connection $\nabla$ and $\boldsymbol{\tau}=\rho \widetilde{V} \otimes \widetilde{V}+\boldsymbol{\tau}_{\text {Max }}$ is given in terms of the stress-energy tensor $\boldsymbol{\tau}_{\text {Max }}$ of the Maxwell 2-form $F$. The Hodge map associated with $\mathbf{g}$ is denoted by $*$. The electric current 3-form $j=*\left(\rho_{e} \widetilde{V}\right)$ is specified in terms of the charge density $\rho_{e}=\frac{e}{m} \rho$ of the fluid with 1-form velocity $\widetilde{V}$. Since $\nabla$.Ein $=0$ and $\mathrm{d} j=0$ it follows from Eq. (13) that $\nabla \cdot \boldsymbol{\tau}=0$ or

$$
\nabla_{V} \widetilde{V}=-\frac{e}{m} i_{V} F
$$

Thus each integral curve of the vector field $V$ models the world line of an electrically charged fluid element under the influence of the Lorentz force. The gravitational forces are encoded into the Levi-Civita connection and are determined by the metric tensor alone.

In a non-Riemannian theory of gravitation the additional torsion and metric gradient fields are expected to provide additional forces, the nature of which depend on the field equations.

4. Divergence of the generalised Einstein tensor. Suppose the non-Riemannian Einstein field equations are given by Eq. (13) for some general stress-energy tensor $\boldsymbol{\tau}$ where

$$
\operatorname{Ein} \equiv \widehat{\mathbf{R i c}}-\frac{1}{2} \mathbf{g} \mathcal{R}
$$

is given in terms of the Ricci tensor $\mathbf{R i c}(X, Y)=\mathbf{R}\left(X_{a}, X, Y, e^{a}\right)$ by

$$
\widehat{\operatorname{Ric}}(X, Y)=\frac{1}{2}(\boldsymbol{R i c}(X, Y)+\operatorname{Ric}(Y, X)) .
$$


Unlike the Einstein tensor associated with the Levi-Civita connection, the Einstein tensor Ein defined in Eq. (17) is associated with the non-Riemannian connection and is not in general divergenceless:

$$
(\nabla \cdot \operatorname{Ein})\left(X_{b}\right) \equiv\left(\nabla_{X_{a}} \operatorname{Ein}\right)\left(X^{a}, X_{b}\right) \neq 0 .
$$

The departure from zero should be expressible in terms of the non-Riemannian fields $\mathbf{T}, \mathbf{S}$, contractions of the generalised curvature tensor and their covariant derivatives. Like the electrical Lorentz force in Eq. (16) above such terms may be associated with gravitational forces that in general may produce non-geodesic motion of matter. Thus an indication of the motion of a fluid in a general non-Riemannian gravitational is given by the divergence of Eq. (13):

$$
\nabla . \tau=\nabla . \text { Ein }
$$

where $\boldsymbol{\tau}$ contains the stress-energy tensor of the fluid.

The non-Riemannian forces $\nabla$.Ein may be expressed as follows. Introduce the tensors

$$
\begin{gathered}
\operatorname{ric}(X, Y)=\mathbf{R}\left(X, Y, X_{a}, e^{a}\right) \\
\mathcal{M} \equiv e^{c}\{\underbrace{\mathcal{S}}_{X, Y, X_{c}}\left(\left(\nabla_{X_{c}}\right)(X, Y)-T\left(X_{c}, T(X, Y)\right)\right)\}
\end{gathered}
$$

where $\underbrace{\mathcal{S}}_{X, Y, Z}$ denotes a cyclic sum over $X, Y, Z$. It follows that

$$
\widehat{\operatorname{Ric}}=\mathbf{R i c}-\mathbf{M}
$$

where $\mathbf{M} \equiv \frac{1}{2}(\mathcal{M}-$ ric $)$. For any tensor $\mathcal{T}$ and vector fields $X, Y, Z, U, V, W, \ldots$, define the tensor $\mathcal{T}(X, Y)$ by

$$
(\mathcal{T}(X, Y))(U, W, \ldots) \equiv \mathcal{T}(X, Y, U, W, \ldots) .
$$

Furthermore for any vector $X$ denote its metric dual by $\tilde{X}$ where $\tilde{X}=\mathbf{g}(X,-)$. Similarly for any 1 -form $\beta$ denote its metric dual by $\widetilde{\beta}$. In terms of this notation one first computes

$$
\left(\nabla_{X_{a}} \widehat{\mathbf{R i c}}\right)\left(X^{a}, X_{b}\right) \equiv(\nabla . \widehat{\mathbf{R i c}})\left(X_{b}\right)=(\nabla \cdot \mathbf{R i c}-\nabla \cdot \mathbf{M})\left(X_{b}\right)
$$

From the definitions above one finds

$$
(\nabla . \text { Ric })\left(X_{b}\right)=\frac{1}{2} X_{b} \mathcal{R}+\mathcal{B}\left(X_{b}\right)
$$

where

$$
\begin{aligned}
\mathcal{B}(X) \equiv & \frac{1}{2} \mathbf{S}\left(X, X^{b}, X^{c}\right) \mathbf{R i c}\left(X_{c}, X_{b}\right)-2\left(\nabla_{X_{a}} \mathbf{M}\right)\left(X, X^{a}\right) \\
& +\frac{1}{2} \mathcal{V}\left(X_{a}, X, X_{\gamma}, X^{b}, e^{a}\right)-\frac{1}{2} \mathcal{V}\left(X, X_{a}, X_{b}, X^{a}, e^{b}\right)
\end{aligned}
$$

and

$$
\begin{aligned}
\mathcal{V}(W, X, Y, Z, \beta) \equiv & \mathbf{R}(X, Y, \widetilde{\beta}, \mathbf{S}(W, Z,-))-\mathbf{R}(X, Y, \widetilde{\mathbf{S}}(W, \beta,-), \widetilde{Z}) \\
& -\mathcal{W}(X, Y, Z, \widetilde{\mathbf{S}}(W, \beta,-))+\left(\nabla_{W} \mathcal{W}\right)(X, Y, Z, \widetilde{\beta})
\end{aligned}
$$

where

$$
\mathcal{W}(U, W) \equiv\left(\nabla_{U} \mathbf{S}\right)(W)-\left(\nabla_{W} \mathbf{S}\right)(U)+\mathbf{S}(T(U, W))
$$


In terms of $\nabla \cdot \mathbf{M} \equiv\left(\nabla_{X_{a}} \mathbf{M}\right)\left(X^{a},-\right)$, it follows that

$$
\nabla . \widehat{\mathbf{R i c}}=\frac{1}{2} \mathrm{~d} \mathcal{R}+\mathcal{B}-\nabla \cdot \mathbf{M}
$$

where

$$
\mathcal{R} \equiv \operatorname{Ric}\left(X_{a}, X^{a}\right) .
$$

Next one calculates $\nabla \cdot(\mathbf{g} \mathcal{R})=\left(\nabla_{X_{a}}(\mathbf{g} \mathcal{R})\right)\left(X^{a},-\right)$. Since

$$
\nabla_{X_{a}}(\mathbf{g} \mathcal{R})=\mathcal{R} \mathbf{S}\left(X_{a},-,-\right)+X_{a} \mathcal{R} \mathbf{g}
$$

then

$$
\nabla \cdot(\mathbf{g} \mathcal{R})=\mathcal{R} \mathbf{S}\left(X_{a}, X^{a},-\right)+\mathrm{d} \mathcal{R} .
$$

Putting together Eq. (30) and Eq. (33) one obtains

$$
\nabla . \text { Ein }=\mathcal{B}-\nabla . \mathbf{M}-\frac{1}{2} \mathcal{R} \mathbf{S}\left(X_{a}, X^{a},-\right) .
$$

Inserting Eq. (34) in Eq. (20) yields an equation of motion for the fluid leading to an interpretation of $\nabla$.Ein in terms of forces on the matter described by $\tau$.

5. Non-Riemannian actions. To gain further insight into the nature of these nonRiemannian forces one must exploit the remaining field equations that determine the torsion and metric gradients. These are most naturally derived from an action principle in which the metric, components of the connection and matter fields are the configuration variables. One requires that an action be stationary with respect to suitable variations of such variables. If the action $n$-form $\Lambda(\mathrm{g}, \nabla, \cdots)$ in $n$ dimensions contains the EinsteinHilbert form

$$
\Lambda_{E H}(\mathbf{g}, \nabla)=\mathcal{R} * 1
$$

and $\underbrace{\Lambda_{E H}}_{\mathbf{g}}$ denotes the variational derivative of $\Lambda_{E H}(\mathbf{g}, \nabla)$ with respect to $\mathbf{g}$ then

$$
\underbrace{\dot{\Lambda_{E H}}}_{\mathbf{g}}=-h^{a b} \operatorname{Ein}\left(X_{a}, X_{b}\right) * 1
$$

where $h_{a b} \equiv \dot{\mathbf{g}}\left(X_{a}, X_{b}\right)$. It is instructive to decompose the non-Riemannian Einstein tensor into parts that depend on the Levi-Civita connection $\stackrel{\circ}{\nabla}$. For this purpose introduce the tensor $\boldsymbol{\lambda}$ by

$$
\boldsymbol{\lambda}(X, Y, \beta)=\beta\left(\nabla_{X} Y\right)-\beta\left(\stackrel{\circ}{\nabla}_{X} Y\right)
$$

for arbitrary vector fields $X, Y$ and 1-form $\beta$. In terms of the exterior covariant derivative $\stackrel{\circ}{\mathrm{D}}$ and Ricci tensor Ric associated with the Levi-Civita connection one may write:

$$
\boldsymbol{\operatorname { R i c }}\left(X_{a}, X_{b}\right)=\operatorname{Ric}\left(X_{a}, X_{b}\right)+i_{a} i_{c}\left(\stackrel{\circ}{\mathrm{D}} \lambda^{c}{ }_{b}+\lambda^{c}{ }_{d} \wedge \lambda^{d}{ }_{b}\right)
$$

where $\lambda^{a}{ }_{b} \equiv \boldsymbol{\lambda}\left(-, X_{b}, e^{a}\right)$ is a set of local 1-forms. It follows from Eqn.17 that Ein differs from the Levi-Civita Einstein tensor Ein by terms involving the tensor $\boldsymbol{\lambda}$ and its derivatives. However for a large class of actions containing the torsion and metric gradient fields one finds that these terms can be dramatically simplified.

To see this simplification most easily it is preferable to change variables from $\mathbf{g}, \nabla$ to $\mathbf{g}, \boldsymbol{\lambda}$ in the total action $n$-form and write $\Lambda(\mathbf{g}, \nabla, \cdots)=\mathcal{L}(\mathbf{g}, \boldsymbol{\lambda}, \cdots)$. Since $\stackrel{\circ}{\nabla}$ depends 
only on the metric it follows from Eqn.37 that $\underbrace{\dot{\lambda}}_{\nabla}=\dot{\nabla}$ and $\underbrace{\dot{\lambda}}_{\mathbf{g}}=-\underbrace{\dot{\dot{\nabla}}}_{\mathbf{g}}$. Hence the variational field equations are

$$
\begin{gathered}
\underbrace{\dot{\Lambda}}_{\nabla}=\underbrace{\dot{\mathcal{L}}}_{\boldsymbol{\lambda}}=0 \\
\underbrace{\dot{\Lambda}}_{\mathrm{g}}=\underbrace{\dot{\mathcal{L}}}_{\mathrm{g}}+\underbrace{\dot{\lambda}}_{\mathrm{g}} \underbrace{\dot{\mathcal{L}}}_{\boldsymbol{\lambda}}=0
\end{gathered}
$$

From Eqn.39 one sees that the term $\underbrace{\dot{\mathcal{L}}}_{\boldsymbol{\lambda}}$ in Eqn.40 does not contribute. To evaluate these variations one first expresses the action in terms of $\mathbf{g}$ and the components of $\boldsymbol{\lambda}$ and its derivatives. To this end it is convenient to introduce the (traceless) 1-forms $\widehat{\lambda}^{a}{ }_{b} \equiv$ $\lambda^{a}{ }_{b}-\frac{1}{n} \delta^{a}{ }_{b} \lambda^{d}{ }_{d}$, and the (traceless) 0 -forms $\widehat{\lambda}^{a}{ }_{b c} \equiv \lambda^{a}{ }_{b c}-\frac{1}{n} \delta^{a}{ }_{b} \lambda^{d}{ }_{d c}$ where $\lambda^{a}{ }_{b c} \equiv i_{c} \lambda^{a}{ }_{b}$. If we express the total action $n$-form $\mathcal{L}(\mathrm{g}, \boldsymbol{\lambda}, \cdots)$ as

$$
\mathcal{L}(\mathbf{g}, \boldsymbol{\lambda}, \cdots)=\mathcal{L}_{E H}(\mathbf{g}, \boldsymbol{\lambda})+\mathcal{F}(\mathbf{g}, \boldsymbol{\lambda}, \cdots)
$$

for some form $\mathcal{F}(\mathrm{g}, \boldsymbol{\lambda}, \cdots)$ where

$$
\mathcal{L}_{E H}=\stackrel{\circ}{\mathcal{R}}-\widehat{\lambda}^{a}{ }_{c} \wedge \widehat{\lambda}^{c}{ }_{b} \wedge *\left(e^{b} \wedge e_{a}\right)-\mathrm{d}\left(\widehat{\lambda}^{a}{ }_{b} \wedge *\left(e^{b} \wedge e_{a}\right)\right)
$$

in terms of the Levi-Civita scalar curvature $\stackrel{\circ}{\mathcal{R}}$ then

$$
\underbrace{\dot{\mathcal{L}_{E H}}}_{\mathbf{g}}=-h^{a b} \operatorname{Ein}\left(X_{a}, X_{b}\right) * 1-h^{a b} \mathcal{E}_{a b} \quad(\bmod \mathrm{d})
$$

where

$$
\mathcal{E}_{a b}=\frac{1}{2} \widehat{\lambda}^{q}{ }_{d} \wedge \widehat{\lambda}^{d}{ }_{p} \wedge\left\{g_{a b} *\left(e^{p} \wedge e_{q}\right)-\delta^{p}{ }_{a} *\left(e_{b} \wedge e_{q}\right)-\delta^{p}{ }_{b} *\left(e_{a} \wedge e_{q}\right)\right\} .
$$

Thus the Einstein field equation is

$$
\operatorname{Ein}\left(X_{a}, X_{b}\right) * 1+\mathcal{E}_{a b}+\mathcal{T}_{a b}=0
$$

where $\underbrace{\dot{\mathcal{F}}}_{\mathbf{g}}=-h^{a b} \mathcal{T}_{a b}$. Next the variations with respect to $\boldsymbol{\lambda}$ yield

$$
\begin{aligned}
& \underbrace{\dot{\mathcal{L}_{E H}}}_{\boldsymbol{\lambda}}=\dot{\widehat{\lambda}}^{a}{ }_{b} \wedge\left\{\hat{\lambda}^{c}{ }_{a} \wedge *\left(e^{b} \wedge e_{c}\right)-\widehat{\lambda}^{b}{ }_{c} \wedge *\left(e^{c} \wedge e_{a}\right)\right\} \quad(\bmod \mathrm{d}) \\
& \underbrace{\dot{\mathcal{F}}}_{\boldsymbol{\lambda}}=\dot{\lambda}^{a}{ }_{b} \wedge \mathcal{F}^{b}{ }_{a} .
\end{aligned}
$$

By splitting off the trace part of the resulting field equation one may write:

$$
\begin{gathered}
\mathcal{F}^{a}{ }_{a}=0 \\
{\widehat{\lambda^{c}}}^{c}{ }_{a} \wedge *\left(e^{b} \wedge e_{c}\right)-\widehat{\lambda}^{b}{ }_{c} \wedge *\left(e^{c} \wedge e_{a}\right)+\widehat{\mathcal{F}}^{b}{ }_{a}=0
\end{gathered}
$$

where $\widehat{\mathcal{F}}^{a}{ }_{b} \equiv \mathcal{F}^{a}{ }_{b}-\frac{1}{n} \delta^{a}{ }_{b} \mathcal{F}^{c}{ }_{c}$. To illustrate how the field equations Eqn.48 and Eqn.49 can greatly simplify the terms in Eqn.44 consider the eight parameter class of models in which the torsion and metric gradient fields enter the action according to:

$$
\begin{aligned}
\mathcal{F} & =4 \sigma R_{a}^{a} \wedge * R_{b}^{b}-2 \ell * 1+\alpha_{1} Q \wedge * Q \\
& +\alpha_{2} u \wedge * u+\alpha_{3} v \wedge * v+\alpha_{4} Q \wedge * u+\alpha_{5} Q \wedge * v+\alpha_{6} u \wedge * v
\end{aligned}
$$


where $\sigma, \alpha_{k}$ are arbitrary coupling constants and $\ell$ is a cosmological constant. The 1 -forms $u$ and $v$ may be expressed in terms of the torsion forms $T^{a}$ and non-metricity forms $Q_{a b}$ as follows:

$$
\begin{gathered}
u \equiv \lambda^{c}{ }_{a c} e^{a}=T-\frac{1}{2} Q \\
v \equiv \lambda_{a}{ }^{c}{ }_{c} e^{a}=\frac{1}{2} Q-\frac{1}{2} \mathcal{Q}-T
\end{gathered}
$$

where $T \equiv i_{a} T^{a}, Q \equiv Q^{a}{ }_{a}=-2 \lambda^{a}{ }_{a}$ and $\mathcal{Q} \equiv e^{a} i^{b} Q_{a b}$. Furthermore $R^{a}{ }_{a}=-\frac{1}{2} \mathrm{~d} Q$ is proportional to the Weyl field 2 -form $\mathrm{d} Q$. Computing the variational derivatives above one finds that Eqn.48 yields:

$$
\mathrm{d} * \mathrm{~d} Q+\frac{4 n \alpha_{1}-\alpha_{4}-\alpha_{5}}{4 n \sigma} * Q+\frac{2 n \alpha_{4}-2 \alpha_{2}-\alpha_{6}}{4 n \sigma} * u+\frac{2 n \alpha_{5}-2 \alpha_{3}-\alpha_{6}}{4 n \sigma} * v=0
$$

while Eqn.49 implies:

$$
\begin{aligned}
& u=\beta_{1} Q \\
& v=\beta_{2} Q
\end{aligned}
$$

and

$$
\begin{gathered}
\widehat{\lambda}^{a}{ }_{b c}=-\frac{n \beta_{1}+n \beta_{2}+1}{n(n-1)(n-2)} \delta^{a}{ }_{b} i_{c} Q \\
+\frac{2 \beta_{2}-2 \beta_{1}+2 n \beta_{2}+1}{2(n-1)(n-2)} g_{b c} i^{a} Q+\frac{2 \beta_{2}-2 \beta_{1}+2 n \beta_{2}+1}{2(n-1)(n-2)} \delta^{a}{ }_{c} i_{b} Q
\end{gathered}
$$

where

$$
\begin{gathered}
\beta_{1}= \\
\left\{n(n-2)-4(n+2)(n-1)^{2} \alpha_{3} \alpha_{4}+2(n+2)(n-1)^{2} \alpha_{5} \alpha_{6}-2 n(n-1) \alpha_{3}+(4-4 n) \alpha_{4}\right. \\
\left.+2(n-1)\left(n^{2}-2\right) \alpha_{5}+n(n-1) \alpha_{6}\right\}\left\{-2 n^{2}(n-2)-2(n+2)(n-1)^{2} \alpha_{6}{ }^{2}\right. \\
\left.+8(n+2)(n-1)^{2} \alpha_{2} \alpha_{3}+8(n-1) \alpha_{2}+8(n-1) \alpha_{3}-4(n-1)\left(n^{2}-2\right) \alpha_{6}\right\}^{-1} \\
\beta_{2}= \\
\left\{n(n-2)+2(n+2)(n-1)^{2} \alpha_{4} \alpha_{6}-4(n+2)(n-1)^{2} \alpha_{2} \alpha_{5}-2 n(n-1) \alpha_{2}\right. \\
\left.+2(n-1)\left(n^{2}-2\right) \alpha_{4}+(4-4 n) \alpha_{5}+n(n-1) \alpha_{6}\right\}\left\{-2 n^{2}(n-2)-2(n+2)(n-1)^{2} \alpha_{6}{ }^{2}\right. \\
\left.+8(n+2)(n-1)^{2} \alpha_{2} \alpha_{3}+8(n-1) \alpha_{2}+8(n-1) \alpha_{3}-4(n-1)\left(n^{2}-2\right) \alpha_{6}\right\}^{-1} .
\end{gathered}
$$

Substituting Eqn.54 and Eqn.55 into Eqn.53 one now obtains a Proca-type equation in the form

where

$$
\mathrm{d} * \mathrm{~d} Q+\beta_{3} * Q=0
$$

$$
\beta_{3}=\frac{4 n \alpha_{1}-\alpha_{4}-\alpha_{5}}{4 n \sigma}+\frac{2 n \alpha_{4}-2 \alpha_{2}-\alpha_{6}}{4 n \sigma} \beta_{1}+\frac{2 n \alpha_{5}-2 \alpha_{3}-\alpha_{6}}{4 n \sigma} \beta_{2}
$$

From the metric variation of $\mathcal{F}$ one finds

$$
\mathcal{T}_{a b}=\ell g_{a b} * 1+\sigma \tau\left(X_{a}, X_{b}\right) * 1+\widehat{\mathcal{T}}_{a b}
$$

where

$$
\boldsymbol{\tau}=*^{-1}\left\{\beta_{3}\left(i_{a} Q \wedge * i_{b} Q-\frac{1}{2} g_{a b} Q \wedge * Q\right)+i_{a} \mathrm{~d} Q \wedge * i_{b} \mathrm{~d} Q-\frac{1}{2} g_{a b} \mathrm{~d} Q \wedge * \mathrm{~d} Q\right\} e^{a} \otimes e^{b}
$$


and

$$
\widehat{\mathcal{T}}_{a b}=\zeta_{1} g_{a b} Q \wedge * Q+\zeta_{2} i_{a} Q \wedge * i_{b} Q
$$

in terms of $\zeta_{1}=\frac{1}{8}\left\{(n+1)(n-2)+4(n-2)\left(n^{3}+n^{2}-4 n-6\right) \beta_{1} \beta_{2}-\left(2 n^{2}+8-2 n^{3}+\right.\right.$ 8n) $\left.\beta_{2}-\left(24+12 n-4 n^{2}\right) \beta_{2}{ }^{2}+\left(2 n^{3}-2 n^{2}+8\right) \beta_{1}+\left(12 n^{2}-24+4 n\right) \beta_{1}{ }^{2}\right\}(n-1)^{-2}(n+2)^{-2}$ and $\zeta_{2}=-\frac{1}{4}\left\{n-2-8(n+1)(n-2) \beta_{1} \beta_{2}-8 \beta_{2} n-\left(8+4 n^{2}+4 n\right) \beta_{2}^{2}+4 \beta_{1} n^{2}-(8+\right.$ $\left.\left.4 n-4 n^{2}-4 n^{3}\right) \beta_{1}{ }^{2}\right\}(n-1)^{-2}(n+2)^{-2}$. Remarkably $\widehat{\mathcal{T}}_{a b}$ exactly cancels $\mathcal{E}_{a b}$ leaving the Einstein equation in the form

$$
\operatorname{Ein}+\ell \mathbf{g}+\sigma \boldsymbol{\tau}=0
$$

6. Conclusion. The solutions to the field equations derived from the action $\mathcal{L}_{E H}(\mathbf{g}, \boldsymbol{\lambda})+\mathcal{F}$ where $\mathcal{F}$ is given by Eqn.50 may be generated from solutions to the Levi-Civita Einstein-Proca system Eqn.56, Eqn.58 with arbitrary cosmological constant $\ell$. Such a reduction promises considerable simplification in the description of the behaviour of matter in the presence of non-Riemannian gravitational fields.

Acknowledgement The authors are grateful to J Schray for valuable interaction. RWT is grateful to the Human Capital and Mobility Programme of the European Union for partial support. CW is grateful to the Committee of Vice-Chancellors and Principals, UK for an Overseas Research Studentship and to Lancaster University for a School of Physics and Chemistry Studentship and a Peel Award.

\section{References}

[1] H. Weyl, Geometrie und Physik, Naturwissenschaften 19 (1931) 49.

[2] J. Scherk, J. H. Schwarz, Phys. Letts 52B (1974) 347.

[3] T. Dereli, R. W. Tucker, Lett. Class. Q. Grav. 12 (1995) L31.

[4] T. Dereli, M. Önder, R. W. Tucker, Lett. Class. Q. Grav. 12 (1995) L25.

[5] T. Dereli, R. W. Tucker, Class. Q. Grav. 11 (1994) 2575.

[6] R. W. Tucker, C. Wang, Class. Quan. Grav. 12 (1995) 2587.

[7] F. W. Hehl, J. D. McCrea, E. W. Mielke, Y. Ne'eman, Physics Reports, 258 (1995) 1.

[8] F. W. Hehl, E. Lord, L. L. Smalley, Gen. Rel. Grav. 13 (1981) 1037.

[9] P. Baekler, F. W. Hehl, E. W. Mielke, "Non-Metricity and Torsion" in Proc. of 4th Marcel Grossman Meeting on General Relativity, Part A, Ed. R Ruffini (North Holland 1986) 277.

[10] V. N. Ponomariev, Y. Obukhov, Gen. Rel. Grav. 14 (1982) 309.

[11] J. D. McCrea, Clas. Q. Grav. 9 (1992) 553.

[12] R. Tresguerres, Z. für Physik C 65 (1995) 347.

[13] R. Tresguerres, Phys.Lett. A200 (1995) 405.

[14] Y. Obukhov, E. J. Vlachynsky, W. Esser, R. Tresguerres, F. W. Hehl, An exact solution of the metric-affine gauge theory with dilation, shear and spin charges, gr-qc 9604027 (1996).

[15] E. J. Vlachynsky, R. Tresguerres, Y. Obukhov, F. W. Hehl, An axially symmetric solution of metric-affine gravity, gr-qc 9604035 (1996).

[16] P. Teyssandier, R. W. Tucker, Class. Quantum Grav 13 (1996) 145. 
[17] T. Dereli, M. Önder, J. Schray, R. W. Tucker, C. Wang, Non-Riemannian Gravity and the Einstein-Proca System, gr-qc 9604039 (1996).

[18] Y. Obhukov, R. Tresguerres, Phys. Lett. A184 (1993) 17.

[19] Y. Ne'eman, F. W. Hehl, Test Matter in a Spacetime with Nonmetricity, gr-qc 9604047 (1996).

[20] H. Kleinert, A. Pelster, Lagrangian Mechanics in Spaces with Curvature and Torsion, gr-qc 9605028 (1996). 